\title{
SOME ASPECTS OF THE DEVELOPMENT OF THE PRONY FILTERING METHOD
}

\author{
Igor B. de Oliveira ${ }^{1}$ iD, Georgy M. Mitrofanov ${ }^{2}$ iD, Viatcheslav I. Priimenko ${ }^{1,3}$ (D), \\ João Carlos P. S. Freires ${ }^{1}$ iD
}

\begin{abstract}
Decomposition based on the Prony transform is widely used to solve various engineering and scientific problems, representing a discrete dataset in the form of a linear combination of complex exponentials or damped sinusoids. Each of these functions is determined by four real parameters: amplitude, attenuation, frequency and phase. In this article, we propose a new workflow, based on the classic Prony and Matrix Pencil methods to determine the damped signals considering their locality and frequency heterogeneity. After estimating the parameters a certain procedure is constructed. This procedure selects the parameters using different criteria and can be called as Prony filtering. We have demonstrated the capabilities of the proposed algorithms and workflow, which provide an optimal set of damped terms for the Prony decomposition for an arbitrary window. The results obtained show good accuracy in the selection of the decomposition components and posterior approximation of damped signals. The accuracy of the results is guaranteed by the quasi-orthogonality of the basis functions of the Prony decomposition on finite intervals.
\end{abstract}

Keywords: Prony's method; Matrix Pencil method; Damped signals; Signal fitting.

RESUMO. A decomposição baseada na transformada de Prony é amplamente utilizada na solução de vários problemas de engenharia e científicos, representando um conjunto de dados discretos na forma de uma combinação linear de exponenciais complexas ou sinusóides amortecidas. Cada uma dessas funções é determinada por quatro parâmetros reais: amplitude, atenuação, frequência e fase. Neste artigo, propomos um novo fluxo de trabalho, baseado nos métodos clássico de Prony e Matrix Pencil, para determinar os sinais amortecidos considerando sua localização e heterogeneidade de frequência. Após estimar os parâmetros, um determinado procedimento é construído. Este procedimento seleciona os parâmetros usando critérios diferentes e pode ser chamado filtragem de Prony. Demonstramos as capacidades dos algoritmos e do fluxo de trabalho propostos, que fornecem um conjunto ótimo de termos amortecidos para a decomposição de Prony em uma janela arbitrária. Os resultados obtidos mostram boa precisão na seleção dos componentes da decomposição e posterior aproximação dos sinais atenuados. A acurácia dos resultados é garantida pela quase-ortogonalidade das funções de base da decomposição de Prony em intervalos finitos.

Palavras-chave: Método de Prony; Método Matrix Pencil; Sinais amortecidos; Ajuste de sinal. 


\section{INTRODUCTION}

Currently, the Prony transform Prony (1795) and the decomposition built on its basis are well known and widely used in solving various engineering and scientific problems starting from early works; see, for instance, Marple-Jr (1987), Agha (1971), Box and Jenkins (1970), Chuang and Moffatt (1976), Felsen and Marcuvitz (1973), McDonough and Higgins (1968). There are numerous mathematical algorithms that implement this decomposition and allow us to represent discrete data in the form of a linear combination of components, which can be either complex exponentials or damped sinusoids. Each of the components is determined by four real parameters: amplitude, attenuation, frequency and phase. The number of components in a linear combination can be determined by the amount of discrete data. In this case, we obtain an interpolation problem in which, when finding the parameters of the components, we try to approximate the available data as accurately as possible.

Interpolation problems arise when it is required to save data or when we know the model that produced the data and it is necessary to accurately determine the values of the parameters of its components. At the same time, the model is often unknown, and data may contain significant errors. Then it becomes necessary to solve approximation problems. In these tasks, we do not need to approximate the data as accurately as possible. However, it is important that the resulting mathematical model determines the essential components of the real object with which the data are associated. These approximation problems that are of great interest in various applied research when it is necessary to determine the values of the parameters of the studied object or to predict its behavior. Seismic exploration and reservoir characterization are one of these areas.

We can indicate the following specific characteristics of seismic data and tasks that require the use of approximate statements (formulations) and significantly complicate their solution:

- Seismic data are very heterogeneous in the composition of the target signals and interference. Moreover, the shape of the signals is often unknown, and the characteristics of the interference are unknown;

- Target models are known with great inaccuracy. This is due to the incompleteness of knowledge about the processes of interaction, propagating elastic vibrations, with complex objects that have significant scale heterogeneity. Thus, it is necessary to solve problems with some uncertainty;

- Data and models have locality and significant frequency dependence. It can be useful to solve real problems aimed at predicting medium parameters. The importance of locality to solve seismic problems is indicated by the fact that it contributes to the appearance of wavelet analysis; see, for instance, Goupillaud et al. (1984), Grossman and Morlet (1984). Locality and frequency dependence are manifested in one of the essential properties of wavelets, namely, frequency-time resolutions; see Chui (1992).

These features played a large role in the development of the Prony filtering method and its application to solve seismic problems in predicting the characteristics of hydrocarbon deposits; see Soares Filho et al. (2003), Mitrofanov and Priimenko (2011), Mitrofanov and Priimenko (2015), Fomel (2013). In particular, understanding the importance of studying the local features of the observed wave field and the medium, as well as the need to ensure the time-frequency resolution, demanded that we use movable windows when estimating the Prony parameters.

One of the important elements of the modern spectral analysis is to clarify the question of the orthogonality of basis functions. In particular, such a study was carried out by Daubechies (1988) for wavelet expansions, where conditions for the orthogonality of basis functions on a finite interval were determined, making possible to decompose signals according to the corresponding basis functions, as well as organize the process of filtering these signals in in order to separate some of the components.

In this work, we propose a new workflow, based on the classic Prony and Matrix Pencil methods, for the Prony filtering of combined damped signals. The results obtained show stable recovery of the signal components, including the attenuation parameter.

The paper is organized as follows. In Section we discuss several specific characteristics of seismic data and tasks that require for using approximate formulations and that can significantly complicate their solution. Section presents the classic Prony and Matrix Pencil methods. In Section we formulate the new workflow used for applying the Prony filtering. Section presents a synthetic signal composed of three damped sinusoids and also a case of modeled data with noise. In Section we analyze the results of the application of the Prony filtering to recover the Prony parameters of the signal. In Section we present some conclusions, which could be made after analyzing the results obtained in this research. Finally, in Appendix we briefly discuss the quasi-orthogonality of the basis functions used in the Prony decomposition.

\section{PRONY METHOD}

A seismic signal can be presented using the Prony transform as a sum of damped sinusoids that lists four real parameters: amplitude, attenuation, frequency and phase for each sinusoid.

After estimating these parameters, a certain procedure can be constructed. This procedure selects the estimated values based on different criteria and can be called Prony filtering. The Prony filtering is analogous 
to bandpass filtering, built on the basis of the Fourier transform, but instead of using only one parameter, in this case frequency, the Prony filtering also considers any of the parameters listed above; see, for instance, Marple-Jr (1987). There is an important difference between the Prony and Fourier transforms: unlike the Fourier transform, where frequency is regularly sampled, the Prony frequencies can have arbitrary values.

\section{Classic Prony Method}

Assume a time domain signal $x[n]$ with $N$ complex samples $x[1], x[2], \ldots, x[N]$. Prony's classic method fits the data, for example, the seismic trace, with the sum of $M$ complex exponential functions, given by Eqs.(1) or (2).

$y[n]=\sum_{k=1}^{M} A_{k} e^{(n-1)\left(\alpha_{k}+2 \pi i f_{k}\right) \tau_{s}+i \theta_{k}}, \quad n=1,2, \ldots, N$,

where $i^{2}=-1$ and $\tau_{s}$ is the sampling interval. The objective is to estimate the amplitudes of complex exponentials $A_{k}$, damping factors $\alpha_{k}$, frequencies $f_{k}$ and phases $\theta_{k}$. If these parameters are determined correctly, then the original signal fits with a high degree of accuracy.

The discrete signal (1) can be expressed in the following equivalent form

$$
y[n]=\sum_{k=1}^{M} h_{k} z_{k}^{n-1},
$$

where $h_{k}=A_{k} e^{i \theta_{k}}$ and $z_{k}=e^{\left(\alpha_{k}+2 \pi i f_{k}\right) \tau_{s}}$ are complex parameters. The fitting of a designated signal is usually accomplished by minimizing the total squared error over the $N$ data values:

$$
\delta=\sum_{n=1}^{N}(\epsilon[n])^{2}
$$

where

$$
\epsilon[n]=x[n]-y[n]=x[n]-\sum_{k=1}^{M} h_{k} z_{k}^{n-1}
$$

represents the complex error between the original data samples $x[n]$ and the linear approximation $y[n]$; see Marple-Jr (1987) for details. This turns out to be a difficult nonlinear problem, which can be solved by the classic Prony method. If as many data samples are used as there are exponential parameters, then an exact exponential fit to the data may be made.

Consider the $M$-exponent discrete function

$$
x[n]=\sum_{k=1}^{M} h_{k} z_{k}^{n-1} .
$$

The $M$ equations of (3) can be written in a matrix form as

$$
\left(\begin{array}{ccc}
z_{1}^{0} & \cdots & z_{M}^{0} \\
\vdots & \ddots & \vdots \\
z_{1}^{M-1} & \cdots & z_{M}^{M-1}
\end{array}\right) \times\left(\begin{array}{c}
h_{1} \\
\vdots \\
h_{M}
\end{array}\right)=\left(\begin{array}{c}
x[1] \\
\vdots \\
x[M]
\end{array}\right) .
$$

Equation (4) is a linear system in $h_{k}, k=1,2, \ldots, M$, with the Vandermonde matrix, which arises naturally from the polynomial interpolation problem. Prony proposed to define the polynomial that has $z_{k}$ as its roots, see Marple-Jr (1987):

$$
F(z)=\prod_{k=1}^{M}\left(z-z_{k}\right) .
$$

Equation (5) may be represented as

$$
F(z)=\sum_{m=0}^{M} a[m] z^{M-m}
$$

with $a[m] \in \mathbb{C}$ and $a[0]=1$.

Shifting index in Eq. (3) from $n$ to $n-m$, multiplying by $a[m]$ we get

$$
a[m] x[n-m]=a[m] \sum_{k=1}^{M} h_{k} z_{k}^{n-m-1} .
$$

Equation (7) can be modified into

$$
\sum_{m=0}^{M} a[m] x[n-m]=\sum_{k=1}^{M} h_{k} z_{k}^{n-M}\left(\sum_{m=0}^{M} a[m] z_{k}^{M-m-1}\right) .
$$

The right-hand internal summation in Eq. (8) may be recognized as polynomial defined by Eq. (6), evaluated at each of its roots $z_{k}$ yielding the zero result

$$
\sum_{m=0}^{M} a[m] x[n-m]=0 .
$$

Equation (9) is a linear difference equation with the homogeneous solution represented by Eq. (3).

The $M$ equations, representing the exact values of $a[m]$ satisfied to Eq. (9), can be represented as an $M \times$ $M$-matrix

$$
\left(\begin{array}{ccc}
x[M] & \cdots & x[1] \\
\vdots & \ddots & \vdots \\
x[2 M-1] & \cdots & x[M]
\end{array}\right) \times\left(\begin{array}{c}
a_{1} \\
\vdots \\
a_{M}
\end{array}\right)=-\left(\begin{array}{c}
x[M+1] \\
\vdots \\
x[2 M]
\end{array}\right) .
$$

Note that the matrix presented in Eq. (10), viewed as a linear system in $a[m], m=1,2, \ldots, M$, is a Toeplitz matrix. Using Eq. (10) we can separate the set of parameters $h_{k}$ from one of $z_{k}$.

Thus, to find coefficients and exponential parameters of Eq. (1), the Prony method can be implemented in 
three steps; see, for instance, Marple-Jr (1987), Mitrofanov and Priimenko (2011):

1. Solve Eq. (10) to find $F(z)$ coefficients.

2. Solve $F(z)=0$, defined by Eq. (6), to find roots $z_{1}, z_{2}, \ldots, z_{M}$. Thus, attenuation factors $\alpha_{k}$ and frequencies $f_{k}$ are expressed by

$$
\alpha_{k}=\frac{1}{\tau_{s}} \ln \left|z_{k}\right|, \quad f_{k}=\frac{1}{2 \pi \tau_{s}} \arctan \left[\frac{\operatorname{Im} z_{k}}{\operatorname{Re} z_{k}}\right] .
$$

3. Solve Eq. (3). For known $\alpha_{k}, f_{k}$ this system is linear. Therefore, amplitudes $A_{k}$ and phases $\theta_{k}$ are

$$
A_{k}=\left|h_{k}\right|, \quad \theta_{k}=\arctan \left[\frac{\operatorname{Im} h_{k}}{\operatorname{Re} h_{k}}\right] .
$$

In real situations, the number of observed data $N$ usually exceeds the minimum number of points required to obtain an exponential model, i.e., $N>2 M$. Following Marple-Jr (1987), in this case Eq. (9) can be modified to the form

$$
\sum_{m=0}^{M} a[m] x[n-m]=e[n],
$$

where $e[n]$ is an estimate of the linear prediction error and differs from the approximation error $\epsilon[n]$. It can be used to determine the parameters $a[n]$ based on minimizing the overall squared error

$$
E=\sum_{n=M+1}^{N}|e[n]|^{2} .
$$

In the case of the least square error (13), the least squares method can be used to solve the problem of obtaining a complex normal equation with a matrix similar to Eq. (4), namely:

$$
\left(\begin{array}{ccc}
x[M] & \cdots & x[1] \\
\vdots & \ddots & \vdots \\
x[N-1] & \cdots & x[N-M]
\end{array}\right) \times\left(\begin{array}{c}
a_{1} \\
\vdots \\
a_{M}
\end{array}\right)=-\left(\begin{array}{c}
x[M+1] \\
\vdots \\
x[N]
\end{array}\right)
$$

As a rule, the solution of Eq. (14) is not very difficult; see, for instance, Marple-Jr (1987), Osbourne and Smyth (1995) for more details. By analogy with the discrete Fourier spectrum we call $\left\{A_{k}, \alpha_{k}, f_{k}, \theta_{k}\right\}_{k=1}^{k=M}$ as the discrete Prony spectrum $\mathcal{R}_{\tau, T}(M)$, i.e.,

$$
\mathcal{R}_{\tau, T}(M)=\left\{A_{k}, \alpha_{k}, f_{k}, \theta_{k}\right\}_{k=1}^{k=M},
$$

where real parameters $\tau$ and $T$ define a time interval where the Prony decomposition has been made up. Here $\tau$ characterizes the position of analyzed time interval and $T=(N-1) \tau_{s}$ is its total wide with a sampling interval $\tau_{s}$. The parameters $\tau$ and $T$ are impor- tant in the Prony decomposition; see Mitrofanov and Priimenko (2015).

For a detailed description of the Prony method and its modifications see, for instance, Marple-Jr (1987), Osbourne and Smyth (1995), Holmström and Peterson (2002), Potts and Tashe (2010).

\section{Matrix Pencil Method}

Given two matrices $\mathbf{Y}_{1}, \mathbf{Y}_{2} \in \mathbb{C}^{p \times i}$, the matrix set of form $\mathbf{Y}_{2}-\lambda \mathbf{Y}_{1}$ with $\lambda \in \mathbb{C}$ is known as the matrix pencil; see, for instance, Rodríguez et al. (2018). To implement the Matrix Pencil method (MPM), a rectangular Hankel matrix $\mathbf{Y}$ is formed by the sign $x[i]$, where, in this method, $P$ represents the pencil parameter:

$$
\mathbf{Y}=\left(\begin{array}{ccccc}
x[1] & x[2] & \cdots & x[P] & x[P+1] \\
x[2] & x[3] & \cdots & x[P+1] & x[P+2] \\
\vdots & \vdots & \ddots & \vdots & \vdots \\
x[N-P] & x[N-P+1] & \cdots & x[N-1] & x[N]
\end{array}\right) .
$$

The matrix $\mathbf{Y}$ is used to create $\mathbf{Y}_{1}$ and $\mathbf{Y}_{2}$. Thus, the matrix $\mathbf{Y}_{1}$ is constructed by deleting the last column of $\mathbf{Y}$, while $\mathbf{Y}_{2}$ is formed by deleting the first column of $\mathbf{Y}$, i.e.,

$$
\mathbf{Y}_{1}=\left(\begin{array}{cccc}
x[1] & x[2] & \cdots & x[P] \\
x[2] & x[3] & \cdots & x[P+1] \\
\vdots & \vdots & \ddots & \vdots \\
x[N-P] & x[N-P+1] & \cdots & x[N-1]
\end{array}\right)
$$

and

$$
\mathbf{Y}_{2}=\left(\begin{array}{cccc}
x[2] & \cdots & x[P] & x[P+1] \\
x[3] & \cdots & x[P+1] & x[P+2] \\
\vdots & \ddots & \vdots & \vdots \\
x[N-P+1] & \cdots & x[N-1] & x[N]
\end{array}\right)
$$

Thus, $z_{p}$ values can be found from the following expression; see Rodríguez et al. (2018):

$$
z_{p}=\text { Eigenvalues }\left(\mathbf{Y}_{1}^{+} \mathbf{Y}_{2}\right)
$$

where $\mathbf{Y}_{1}^{+}$is the pseudoinverse matrix of $\mathbf{Y}_{1}$, defined as

$$
\mathbf{Y}_{1}^{+}=\left[\mathbf{Y}_{1}^{H} \mathbf{Y}_{1}\right]^{-1} \mathbf{Y}_{1}^{H} \text {. }
$$

Finally, with $z_{p}$ values, the attenuation, frequencies, amplitudes and phases can be calculated according to Eqs. (11)-(12), by changing the index $k$ to $p$.

\section{WORKFLOW}

The new workflow developed in this work for applying the Prony filtering consists of six main steps, as described in Fig. 1. All computational implementations 
were performed using the Python programming language.

The first step of the workflow consists of signal modeling, described in more detail in Section .

Then, the signal is separated into several windows, according to the definition of two parameters: the window size and stride, which regulates the window shift. In this work, a $248 \mathrm{~ms}$ window and a $50 \mathrm{~ms}$ stride were chosen, as an example. Thereafter, for each signal window, the following steps are applied:

- Prony decomposition: this consists of the application of the Matrix Pencil method, described in Section, to estimate the four Prony parameters - attenuation factor, frequency, amplitude and phase. In this work, the number of terms, or $M$ complex exponential functions, was calculated through an optimization process, where for each window the number of terms was defined as the value that minimizes the RMS error between the modeled and the estimated signal;

- Prony approximation: where the signal is approximated according to the parameters estimated during the Prony decomposition;

- Selection of Prony parameters: step where the Prony filtering is applied, by selecting the estimated parameters that most closely match the modeled ones;

- Graph and table elaboration: it constitutes the export of graphs and tables with the results.

Table 1. Parameters used for creating three damped sinusoids

\begin{tabular}{|l|c|c|c|c|}
\hline Sinusoids & Attenuation $\alpha$ & Frequency $f$ & Amplitude $A$ & Phase $\theta$ \\
\hline Sinusoid 1 & -3 & 10 & 1.0 & $-\pi / 2$ \\
\hline Sinusoid 2 & -6 & 25 & 1.7 & 0 \\
\hline Sinusoid 3 & -5 & 15 & 1.3 & $\pi$ \\
\hline
\end{tabular}

\section{SIGNAL MODELING}

Initially, using Eq. (15), three damped sinusoids were created, considering the parameters given in Table 1. Each signal starts its propagation at a different time: 0 $\mathrm{ms}, 100 \mathrm{~ms}$ and $200 \mathrm{~ms}$, respectively, and the time step is $2 \mathrm{~ms}$ :

$$
s(t)=A e^{\alpha t} \cos (2 \pi f t+\theta) .
$$

Then, the sum of the three sinusoids was calculated to obtain the modeled signal (without adding any noise), extending from $0 \mathrm{~ms}$ to $398 \mathrm{~ms}$, as shown in Fig. 2.

Since in the real world we always have a noise component present in the data, we created a second experiment by adding Gaussian noise with zero mean and 0.1 standard deviation, as illustrated in Fig. 3.

\section{RESULTS AND DISCUSSION}

The two modeled signals mentioned above were some of the many experiments performed to test the considered computational methods, in particular, the Matrix Pencil method (MPM). In these experiments, when forming model traces, three types of damped sinusoids were taken with different parameters and time delays from the beginning of the record. This determined the complexity of the model structure with the presence of two discontinuities at times of 100 and $200 \mathrm{~ms}$; see Figs. 2 and 3 . It was interesting to check how the tested method deals with the approximation of such functions.

The length of each time interval was $248 \mathrm{~ms}$, that is the duration of the window with which the corresponding segment of the model trace was cut, as described in Section. The step (or stride) for the windows was taken equal to $50 \mathrm{~ms}$. Thus, we obtained four different windows to cover the entire modeled signal: [0,248], $[50,298],[100,348]$, and $[150,398] \mathrm{ms}$.

Figures 4 and 7 show the results obtained, comparing the modeled signal for each window (dotted curve) and the Prony approximation (continuous curve).

As shown in these figures, especially in the case without noise, using the Matrix Pencil method provides an ideal approximation based on the Prony expansion with zero RMS error, approximately. It can be noted that in all four windows there are features of the simulated bursttype signal. Consequently, it is not the presence of such features that leads to some inaccurate signal approximations, but their location within the analyzed interval. Therefore, changing the window position can improve the accuracy of the approximation.

Let us note one more interesting point revealed during the performance of these experiments. It is concerned with optimizing the number of terms in the Prony approximation that provides the best accuracy. For example, for the first experiment, the best fit was achieved using 54, 56, 54, and 30 terms, respectively, for each of the above windows. This number of complex exponential functions was automatically determined by the method developed and implemented here, through an optimization function, which analyzes the distribution of the RMS error according to the variation of the number of terms.

As presented in the workflow, after the Prony approximation, we performed the selection (or filtering) of the estimated parameters that most closely match the modeled ones through an analysis of the joint absolute error between the Prony parameters, attenuation factor and frequency, modeled and estimated. Figures 5 and 8 show that parameters are given in pairs since we are approximating the real-valued signal using complex exponential functions.

Thus, we apply Prony filtering to the results obtained using tens of terms, selecting only six terms or three pairs of the parameters to reconstruct the modeled ones. Tables 2-9 show the values of these parameters for each window, where, for compactness, the values 


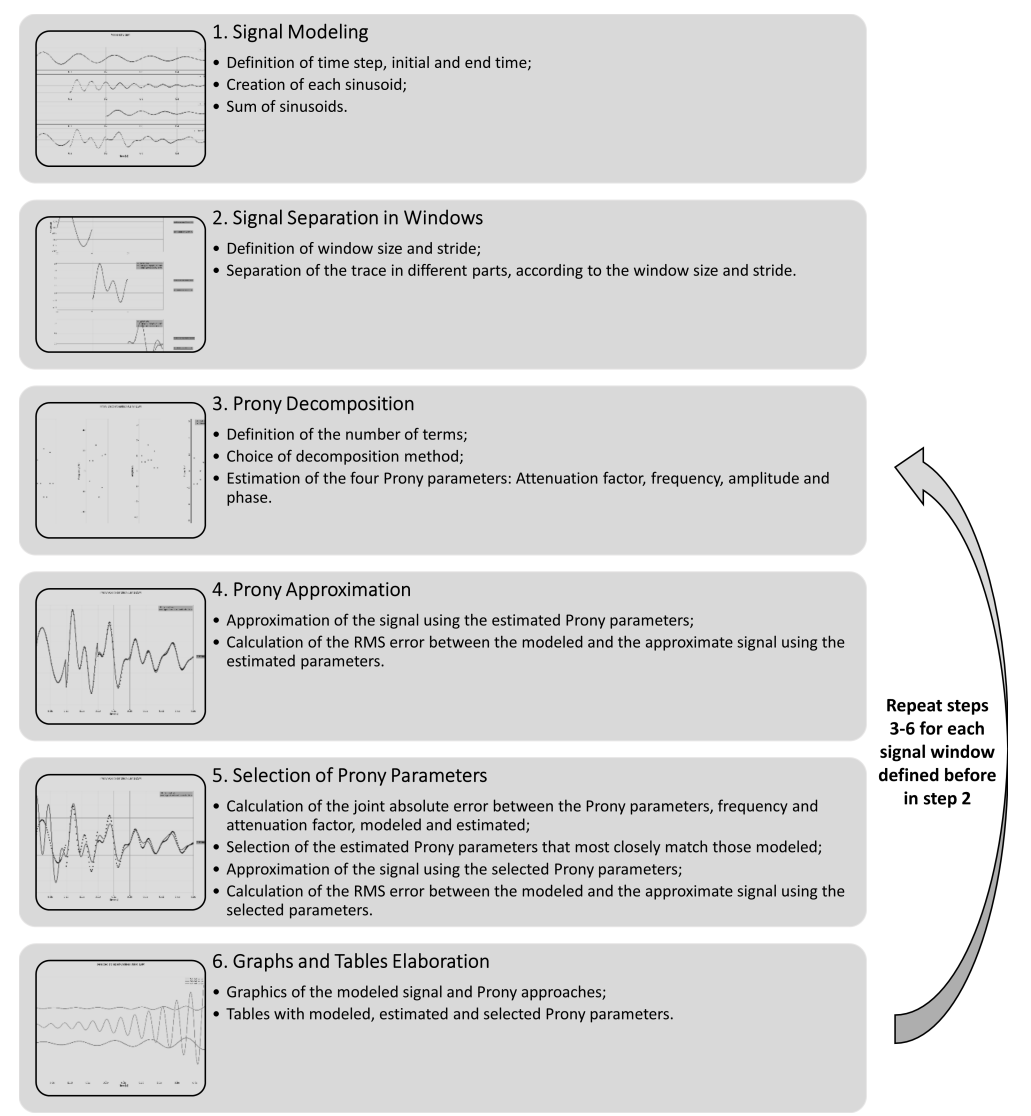

Figure 1. Prony filtering workflow

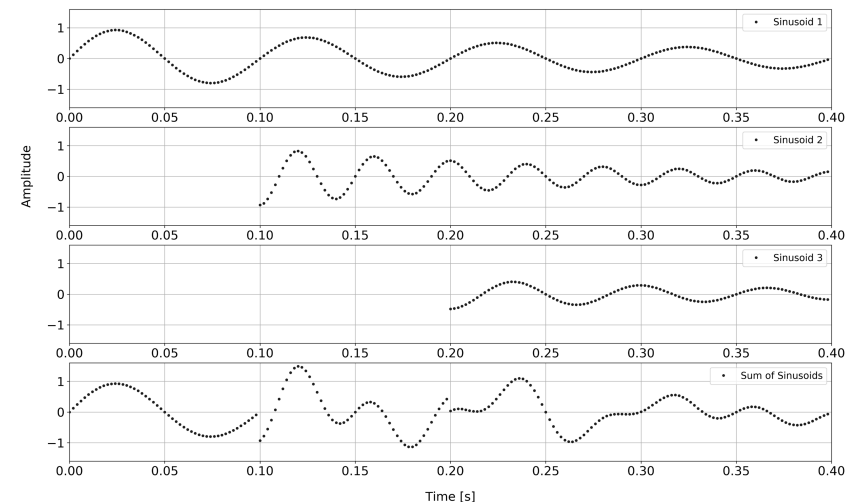

Figure 2. Modeled signal without noise

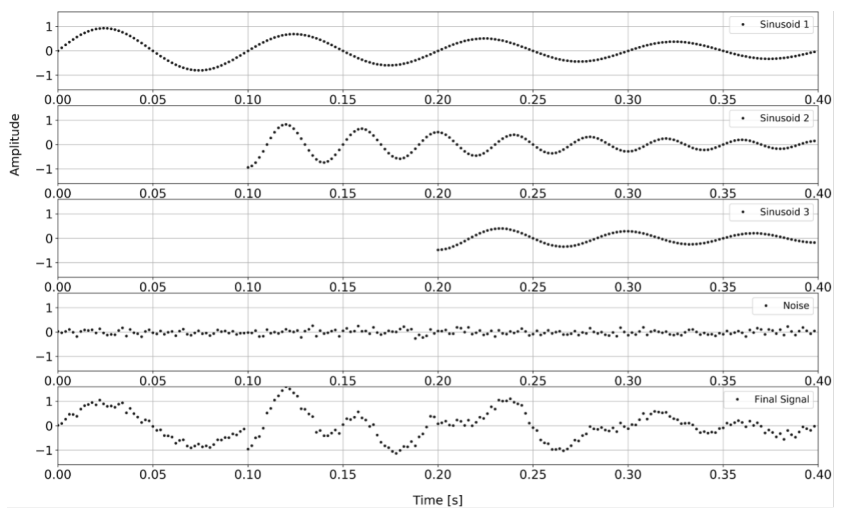

Figure 3. Modeled signal with noise

uation parameter was also estimated with some accuracy for some windows, both for the case without noise and with noise. This difficulty in getting it right can be explained by the time shifts of the modeled sinusoids, which introduce greater complexity in the shape of the signals, with the presence of discontinuities.

The appearance in Tables 2, 3, 6-8 of positive values of the attenuation coefficient is associated with the complexity of the analyzed signal and the position of the moving window on the time axis. As we can see from Tables 4, 5, and 9, in the case when the moving windows are shifted to the right along the time axis, all the attenuation values become negative. frequency parameter was successfully extracted, very close to the simulated values. Furthermore, the atten- 

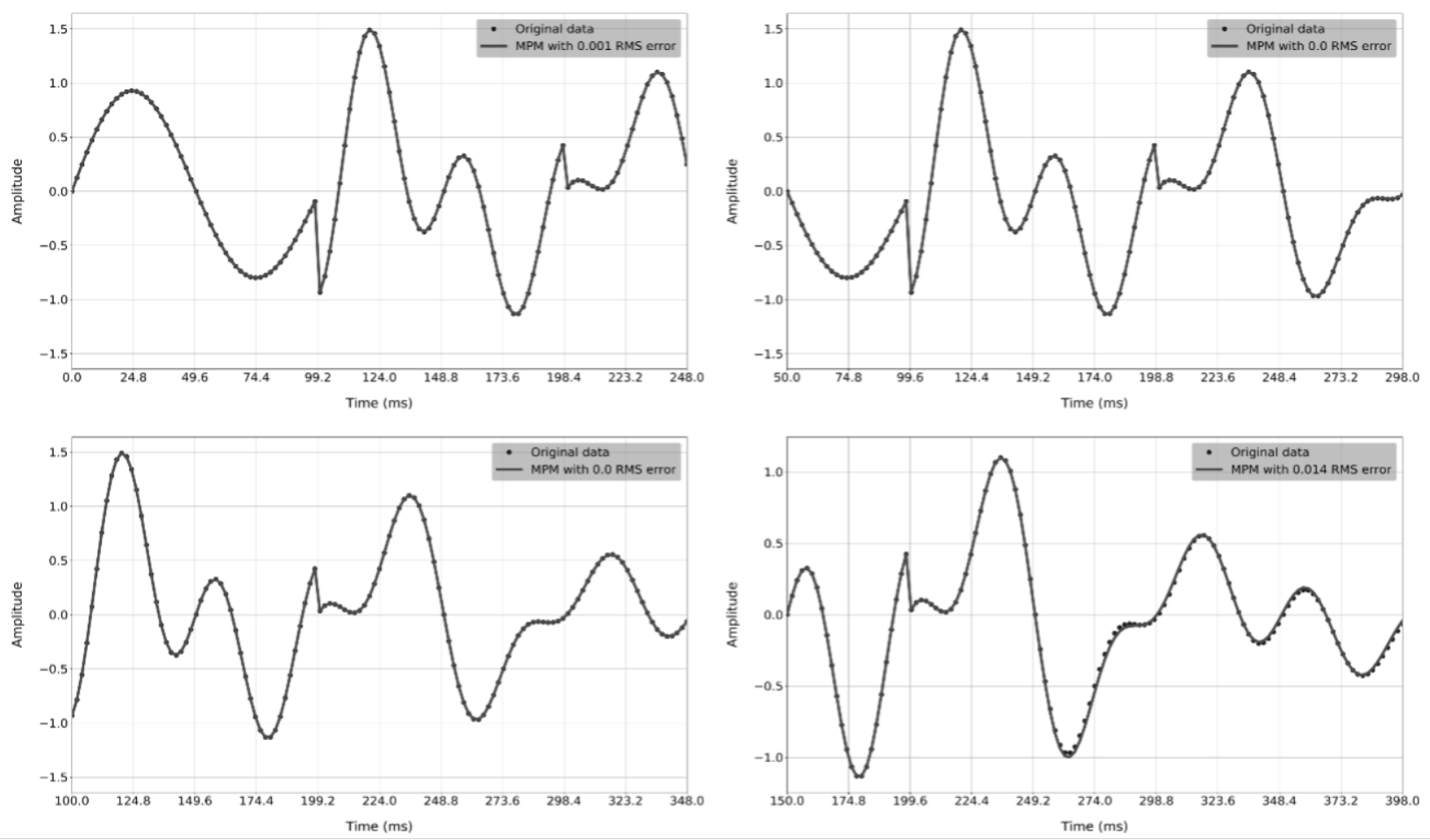

Figure 4. Comparison between modeled signal without noise and approximate signal using the Matrix Pencil method for four different time windows: [0, 248], [50, 298], [100,348] and [150, 398] ms
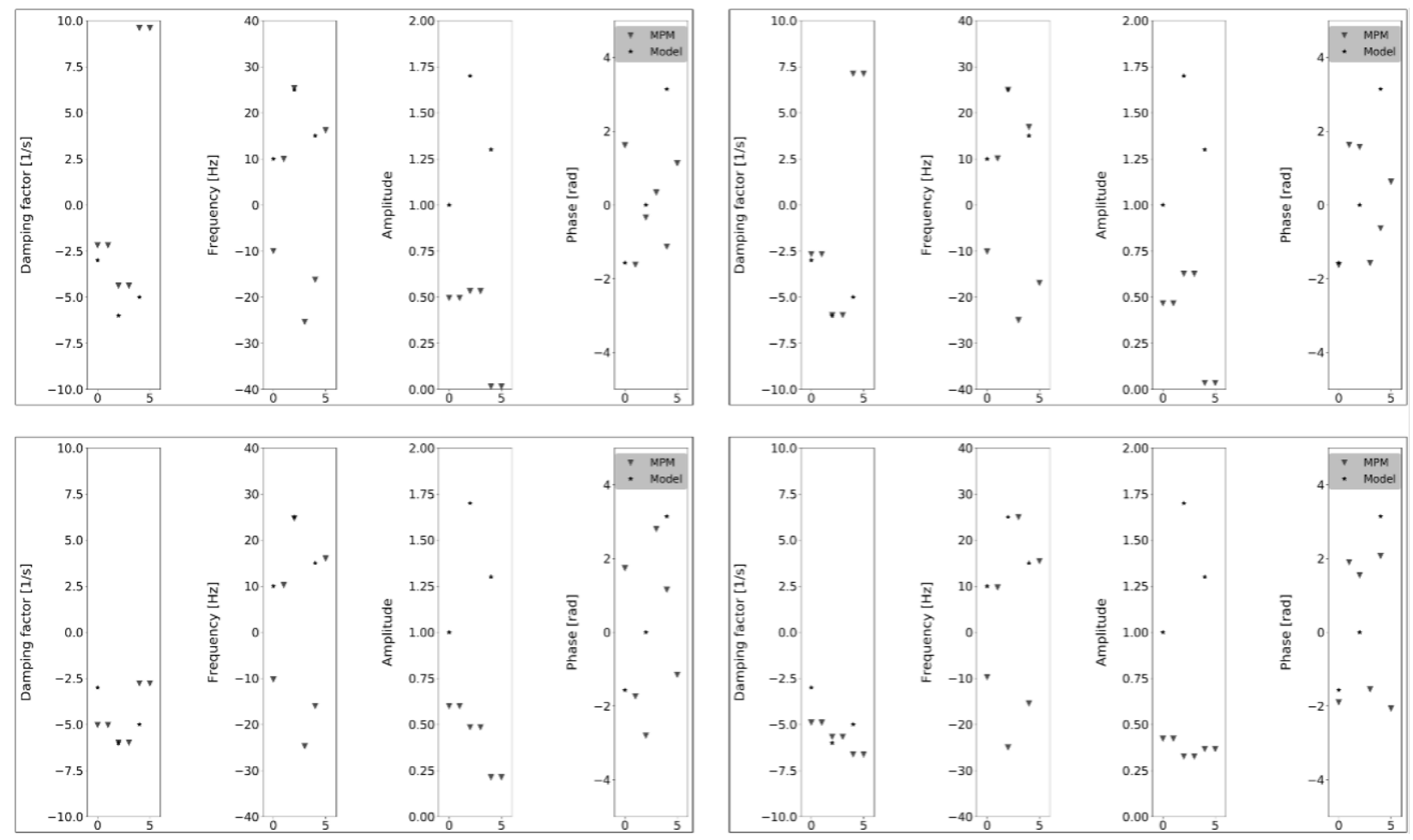

Figure 5. Comparison between the Prony parameters used in signal modeling and those selected from the parameters estimated by the Matrix Pencil method for four different time windows of modeled signal without noise 

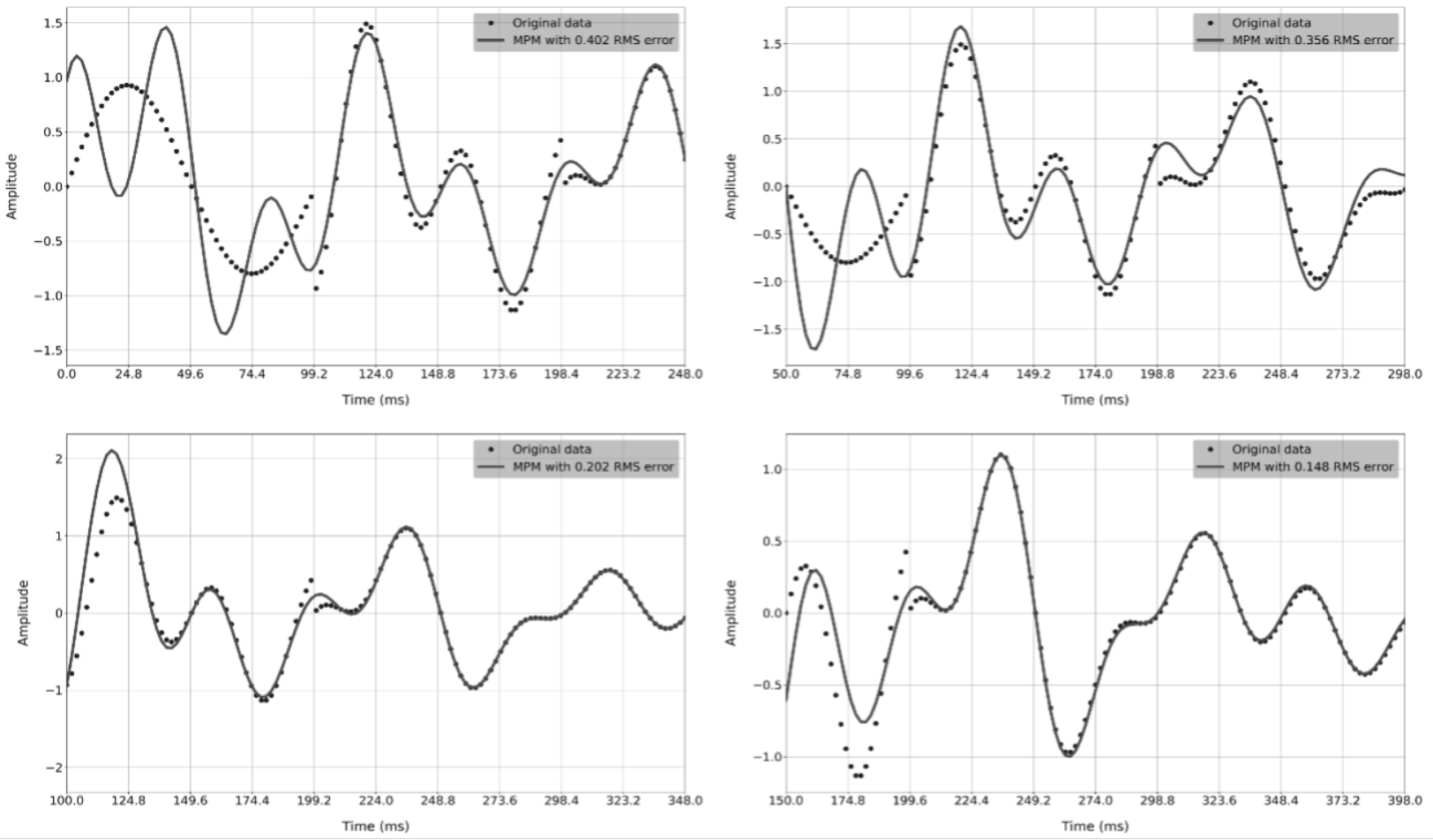

Figure 6. Comparison between modeled signal without noise and approximate signal using only the Prony parameters selected from those estimated by the Matrix Pencil method for four different time windows
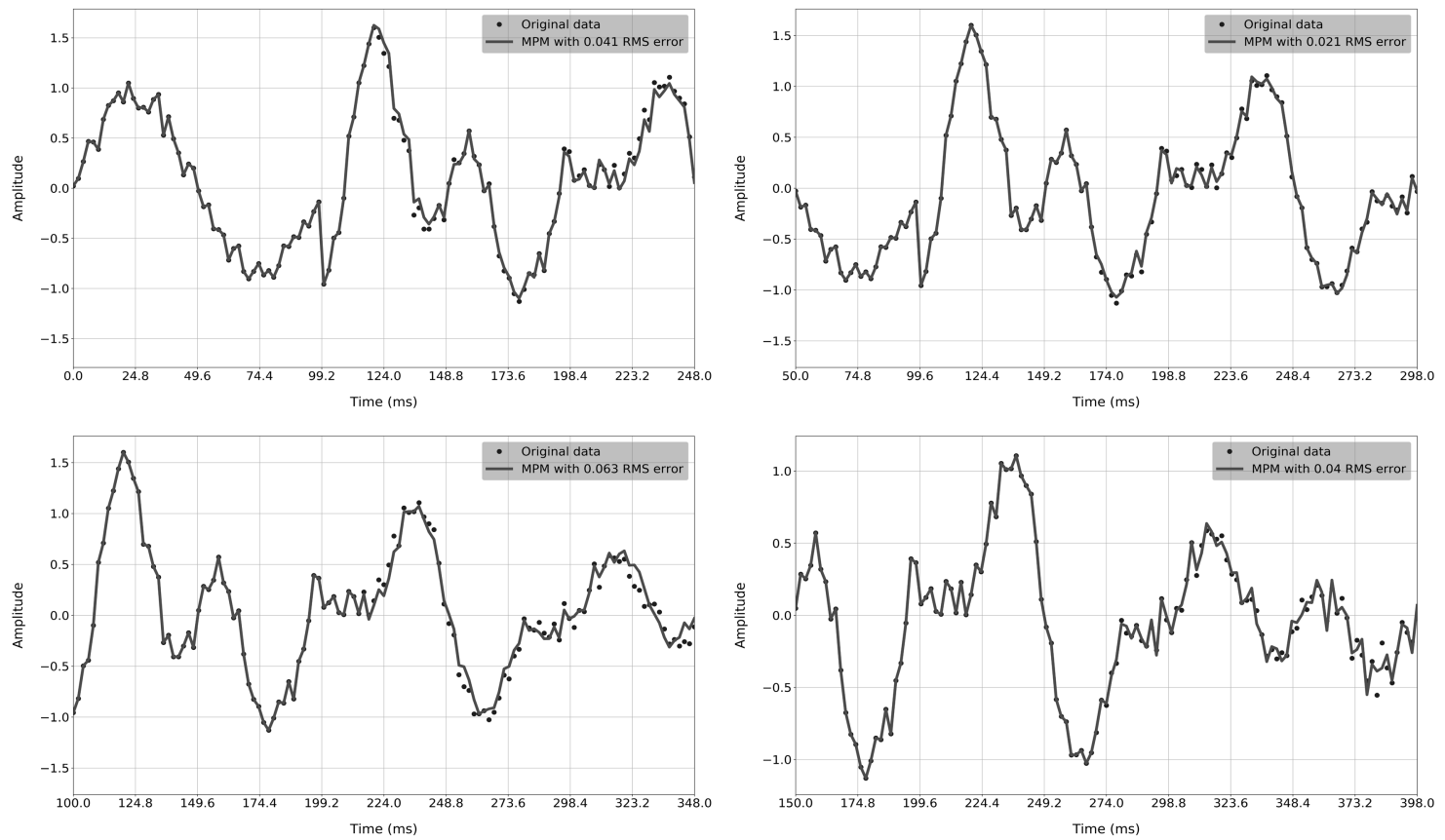

Figure 7. Comparison between modeled signal with noise and approximate signal using the Matrix Pencil method for four different time windows 

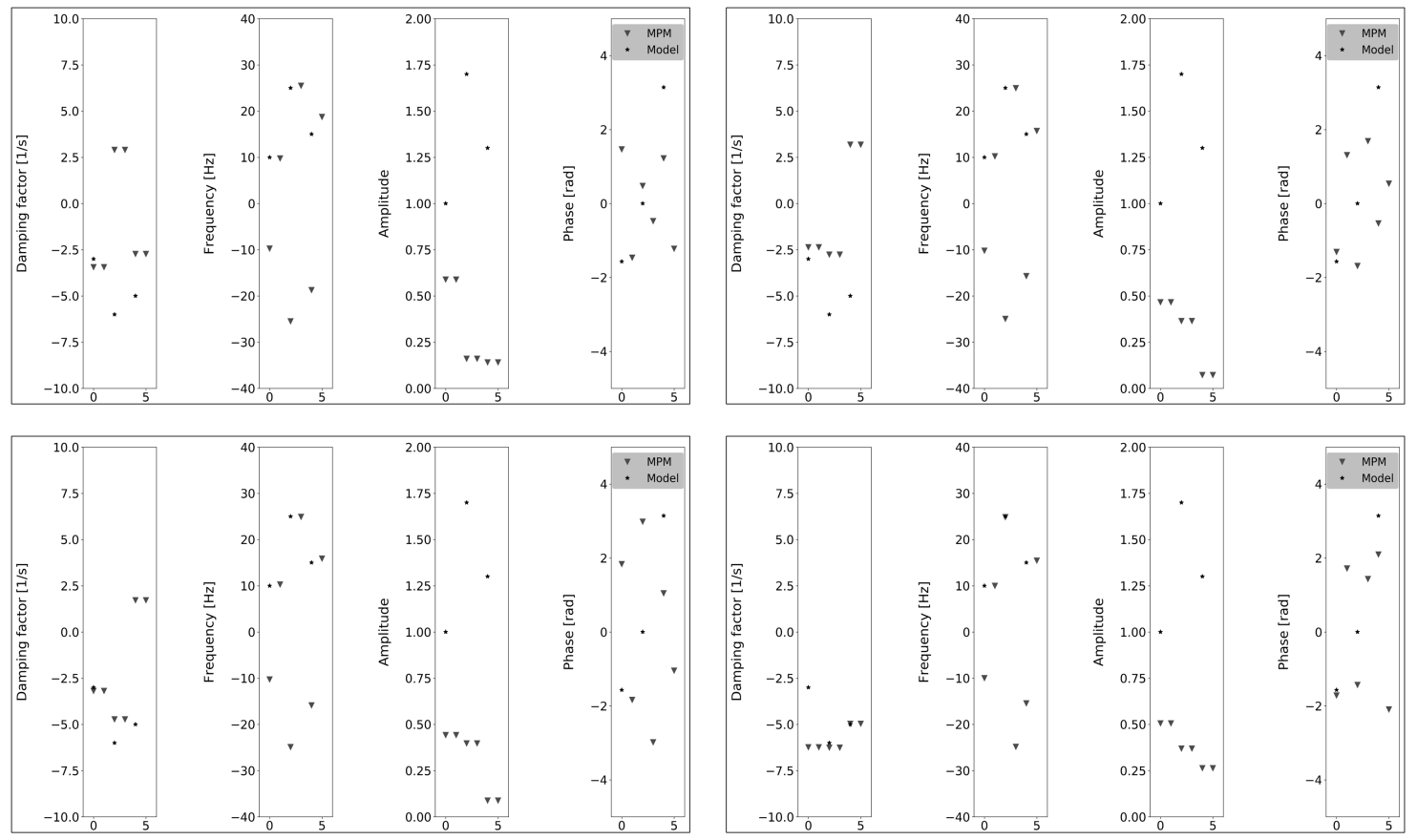

Figure 8. Comparison between the Prony parameters used in signal modeling and those selected from the parameters estimated by the Matrix Pencil method for four different time windows of modeled signal with noise
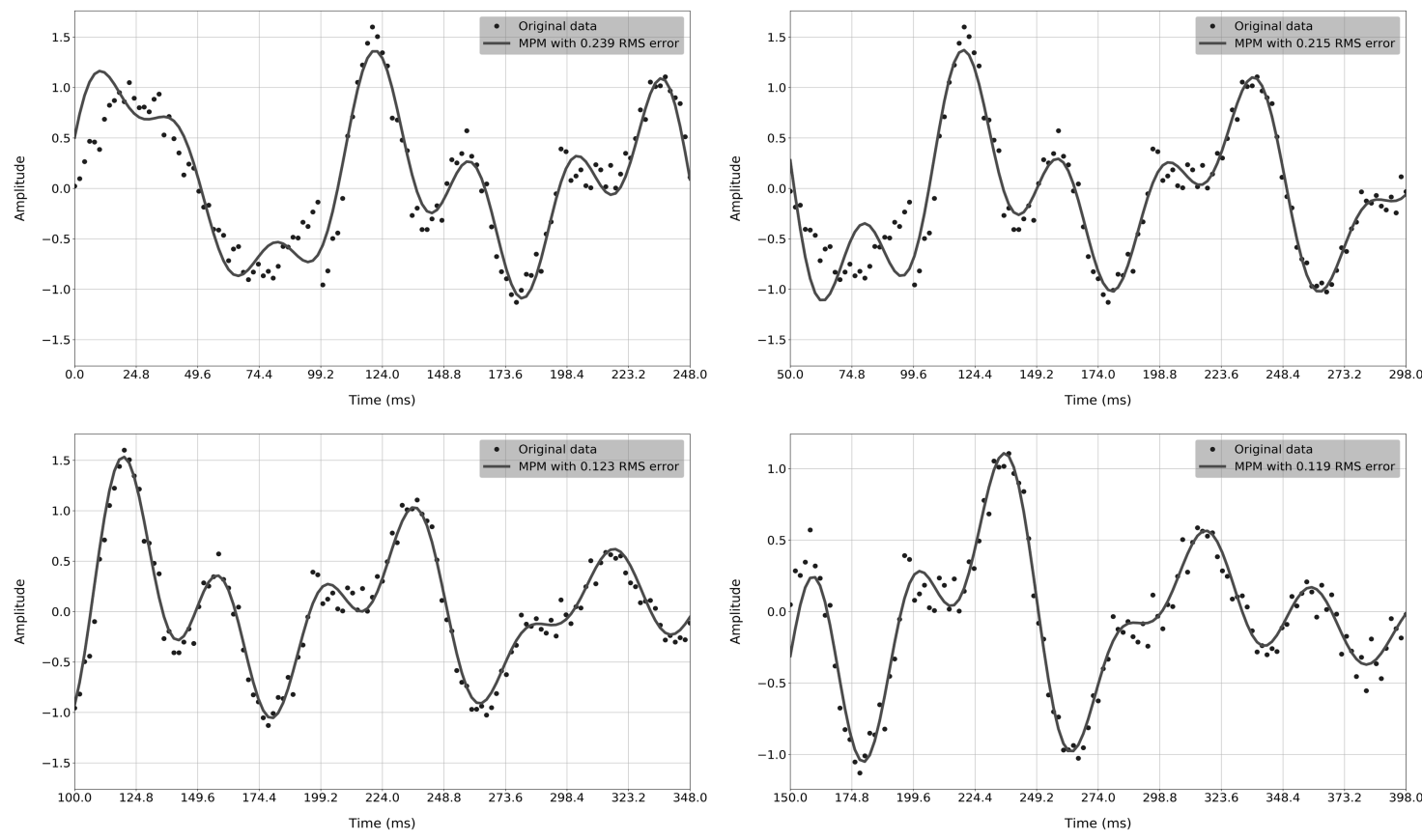

Figure 9. Comparison between modeled signal with noise and approximate signal using only the Prony parameters selected from those estimated by the Matrix Pencil method for four different time windows 
Table 2. Selected parameters for modeled signal without noise, $[0,248] \mathrm{ms}$ window

\begin{tabular}{|c|c|c|c|c|}
\hline Function & Attenuation $\alpha$ & Frequency $f$ & Amplitude $A$ & Phase $\theta$ \\
\hline 1 & -2.18439 & -9.98239 & 0.49583 & 1.62027 \\
\hline 2 & -2.18439 & 9.98239 & 0.49583 & -1.62027 \\
\hline 3 & -4.38270 & 25.35830 & 0.53224 & -0.33859 \\
\hline 4 & -4.38270 & -25.35830 & 0.53224 & 0.33859 \\
\hline 5 & 9.59525 & -16.22191 & 0.01516 & -1.12839 \\
\hline 6 & 9.59525 & 16.22191 & 0.01516 & 1.12839 \\
\hline
\end{tabular}

Table 3. Selected parameters for modeled signal without noise, $[50,298] \mathrm{ms}$ window

\begin{tabular}{|c|c|c|c|c|}
\hline Function & Attenuation $\alpha$ & Frequency $f$ & Amplitude $A$ & Phase $\theta$ \\
\hline 1 & -2.66025 & -10.11340 & 0.46685 & -1.62990 \\
\hline 2 & -2.66025 & 10.11340 & 0.46685 & 1.62990 \\
\hline 3 & -5.97282 & 24.99800 & 0.62669 & 1.57670 \\
\hline 4 & -5.97282 & -24.99800 & 0.62669 & -1.57670 \\
\hline 5 & 7.12121 & 16.91443 & 0.03466 & -0.63073 \\
\hline 6 & 7.12121 & -16.91443 & 0.03466 & 0.63073 \\
\hline
\end{tabular}

Table 4. Selected parameters for modeled signal without noise, [100, 348] ms window

\begin{tabular}{|c|c|c|c|c|}
\hline Function & Attenuation $\alpha$ & Frequency $f$ & Amplitude $A$ & Phase $\theta$ \\
\hline 1 & -5.03235 & -10.24423 & 0.59931 & 1.73967 \\
\hline 2 & -5.03235 & 10.24423 & 0.59931 & -1.73967 \\
\hline 3 & -5.98212 & 24.73287 & 0.48464 & -2.80396 \\
\hline 4 & -5.98212 & -24.73287 & 0.48464 & 2.80396 \\
\hline 5 & -2.76875 & -16.00921 & 0.21379 & 1.15595 \\
\hline 6 & -2.76875 & 16.00921 & 0.21379 & -1.15595 \\
\hline
\end{tabular}

Table 5. Selected parameters for modeled signal without noise, [150, 398] ms window

\begin{tabular}{|c|c|c|c|c|}
\hline Function & Attenuation $\alpha$ & Frequency $f$ & Amplitude $A$ & Phase $\theta$ \\
\hline 1 & -4.90086 & -9.72909 & 0.42292 & -1.90043 \\
\hline 2 & -4.90086 & 9.72909 & 0.42292 & 1.90043 \\
\hline 3 & -5.67434 & -24.99353 & 0.32679 & 1.54622 \\
\hline 4 & -5.67434 & 24.99353 & 0.32679 & -1.54622 \\
\hline 5 & -6.62032 & -15.44908 & 0.36686 & 2.06826 \\
\hline 6 & -6.62032 & 15.44908 & 0.36686 & -2.06826 \\
\hline
\end{tabular}

\section{CONCLUSION}

Modern computational methods provide a good approximation of fairly complex signals using the Prony decomposition. These signals can be the result of the interference of several simple pulses with different Prony parameters and initial times. Meanwhile, when performing such an approximation, a large number of terms
Table 6. Selected parameters for modeled signal with noise, $[0,248] \mathrm{ms}$ window

\begin{tabular}{|c|c|c|c|c|}
\hline Function & Attenuation $\alpha$ & Frequency $f$ & Amplitude $A$ & Phase $\theta$ \\
\hline 1 & -3.44483 & -9.74029 & 0.58894 & 1.46579 \\
\hline 2 & -3.44483 & 9.74029 & 0.58894 & -1.46579 \\
\hline 3 & 2.89959 & -25.48776 & 0.15993 & 0.47543 \\
\hline 4 & 2.89959 & 25.48776 & 0.15993 & -0.47543 \\
\hline 5 & -2.72651 & -18.73164 & 0.14092 & 1.22228 \\
\hline 6 & -2.72651 & 18.73164 & 0.14092 & -1.22228 \\
\hline
\end{tabular}

Table 7. Selected parameters for modeled signal with noise, $[50,298] \mathrm{ms}$ window

\begin{tabular}{|c|c|c|c|c|}
\hline Function & Attenuation $\alpha$ & Frequency $f$ & Amplitude $A$ & Phase $\theta$ \\
\hline 1 & -2.36816 & -10.22075 & 0.46624 & -1.31115 \\
\hline 2 & -2.36816 & 10.22075 & 0.46624 & 1.31115 \\
\hline 3 & -2.76234 & -24.97824 & 0.36447 & -1.68889 \\
\hline 4 & -2.76234 & 24.97824 & 0.36447 & -1.68889 \\
\hline 5 & 3.18433 & -15.70503 & 0.07244 & -0.53802 \\
\hline 6 & 3.18433 & 15.70503 & 0.07244 & 0.53802 \\
\hline
\end{tabular}

Table 8. Selected parameters for modeled signal with noise, [100, 348] ms window

\begin{tabular}{|c|c|c|c|c|}
\hline Function & Attenuation $\alpha$ & Frequency $f$ & Amplitude $A$ & Phase $\theta$ \\
\hline 1 & -3.18666 & -10.27012 & 0.44211 & 1.83448 \\
\hline 2 & -3.18666 & 10.27012 & 0.44211 & -1.83448 \\
\hline 3 & -4.72431 & -24.89936 & 0.39781 & 2.98451 \\
\hline 4 & -4.72431 & 24.89936 & 0.39781 & -2.98451 \\
\hline 5 & 1.71798 & -15.87428 & 0.08820 & 1.04556 \\
\hline 6 & 1.71798 & 15.87428 & 0.08820 & -1.04556 \\
\hline
\end{tabular}

Table 9. Selected parameters for modeled signal with noise, [150, 398] ms window

\begin{tabular}{|c|c|c|c|c|}
\hline Function & Attenuation $\alpha$ & Frequency $f$ & Amplitude $A$ & Phase $\theta$ \\
\hline 1 & -6.23142 & -9.97466 & 0.50574 & -1.71763 \\
\hline 2 & -6.23142 & 9.97466 & 0.50574 & 1.71763 \\
\hline 3 & -6.25736 & 24.84385 & 0.36939 & -1.43325 \\
\hline 4 & -6.25736 & -24.84385 & 0.36939 & 1.43325 \\
\hline 5 & -4.96550 & -15.44495 & 0.26344 & 2.09705 \\
\hline 6 & -4.96550 & 15.44495 & 0.26344 & -2.09705 \\
\hline
\end{tabular}

are used, each of which has its own set of parameters. Among them, there are also target values corresponding to the simulated signals, but they are hidden by the parameters of other components. Due to the peculiarities of the Prony transform, it is the sharp and discontin- 
uous elements of the interference signal that form additional components with high frequency, attenuation, and amplitude. The elimination of such components can be realized by analyzing and selecting the resulting sets of the Prony parameters. This selection we called Prony filtering. Thus, as a result of the Prony filtering, the target components of the analyzed signals can be simultaneously selected and the elements distorting them can be eliminated.

In this paper, we have demonstrated the capabilities of the proposed workflow, which provides an optimal set of conditions to the Prony decomposition for an arbitrary window. In our experiments, we used these windows with different positions concerning the signal of interest. The workflow also made it possible to demonstrate the fundamental possibility of separating individual damped components that form the simulated signal, i.e., the possibility of implementing the Prony filtering procedure.

During the experiments, we noticed that in addition to the window position, the accuracy of approximation and selection of the damped components can be affected by the interval duration, i.e., the window width. Thus, further research is needed to find an optimum window width with the optimization of the number of components and the most accurate determination of the target pulses. This definition will also allow us to select target pulses at an unknown location on the time axis.

\section{ACKNOWLEDGMENTS}

The authors would like to thank the Laboratory of Engineering and Exploration of Petroleum of the State University of Norte Fluminense Darcy Ribeiro, RJ, Brazil, for creating conditions for this work.

\section{APPENDIX A.}

Let us consider one important property of damped sinusoids, which does not depend on the method of their estimation and characterizes the degree of quasiorthogonality of the Prony decomposition components on a finite interval. Equation (1) can be considered as an expansion in basis functions, which are damped cosine waves. According to Marple-Jr (1987), this equation for real-valued signals can be rewritten as

$$
y[n]=\sum_{m=1}^{M} A_{m} e^{\alpha_{m}(n-1) \tau_{s}} \cos \left(2 \pi f_{m}(n-1) \tau_{s}+\theta_{m}\right) .
$$

Then vector $\mathbf{y}=(y[1], y[2], \ldots, y[N])^{*}$, where * means transposition, can be represented as a linear combination of vectors $\mathbf{b}_{m}=\left(b_{m}[1], b_{m}[2], \ldots, b_{m}[N]\right)^{*}$ with $b_{m}[k]$ defined as follows:

$$
b_{m}[k]=e^{\alpha_{m}(k-1) \tau_{s}} \cos \left(2 \pi f_{m}(k-1) \tau_{s}+\theta_{m}\right)
$$

Where $k=1,2, \ldots, N$. Thus, we get

$$
\mathbf{y}=\sum_{m=1}^{M} A_{m} \mathbf{b}_{m}
$$

If vectors $\mathbf{b}_{m}$ are linearly independent, then they can be considered as basis vectors. To make full use of linear algebraic constructions, we need to study two points:

- linear independence of vectors;

- degree of orthogonality of vectors among themselves.

The linear independence of $\mathbf{b}_{m}$ can be determined by considering the corresponding matrix, and the degree of their orthogonality is determined by the values of the angle between these vectors. To simplify this analysis, in Mitrofanov and Priimenko (2015) was made the transition to continuous functions associated with vectors $\mathbf{b}_{m}$. Such transition allowed us to determine the scalar product of the basis vectors in an integral form. As the result, it was possible to construct a general expression for the cosine of the angle between vectors in the following form:

$$
\cos \left(\mathbf{b}_{i} \wedge \mathbf{b}_{j}\right) \cong \frac{I_{i j}^{(1)}}{\sqrt{I_{i}^{(0)} \cdot I_{j}^{(0)}}},
$$

where

$$
\begin{aligned}
& I_{i j}^{(1)}=\int_{0}^{T} e^{\left(\alpha_{i}+\alpha_{j}\right) t} \cos \left(2 \pi f_{i} t+\theta_{i}\right) \cos \left(2 \pi f_{j} t+\theta_{j}\right) d t, \\
& I_{i}^{(0)}=\int_{0}^{T} e^{2 \alpha_{i} t} \cos ^{2}\left(2 \pi f_{i} t+\theta_{i}\right) d t .
\end{aligned}
$$

These integrals are calculated explicitly, which makes it possible to study the relationship between vectors $\mathbf{b}_{m}$ depending on the values of $\alpha, f, \theta, T$, where $T=N \cdot \tau_{s}$ determines the interval for $\mathbf{y}$ and $\mathbf{b}_{m}$. Equation (A1) allows us to estimate the degree of orthogonality of the basis vectors; see Mitrofanov and Priimenko (2015) for more details.

\section{REFERENCES}

Agha, M. A direct method for fitting linear combinations of exponentials. Biometrics 1971, 27, 399-413.

Box, G.; Jenkins, G. Time series analysis forecasting and control; San Francisco, CA: Holden-Day, 1970.

Chuang, C.; Moffatt, D. Natural resonances of radar targets via Prony's method and target discrimination. IEEE Transactions on Aerospace and Electronic Systems, AES 1976, 12, 583-589.

Chui, C. Wavelet Analysis and its Applications. Volume 1: An Introduction to Wavelets; Academic Press Inc., 1992.

Daubechies, I. Orthonormal bases of compactly supported wavelets. Comm. Pure Appl. Math. 1988, 41, 909-996.

Felsen, L.; Marcuvitz, N. Radiation and scattering of waves; Englewood Cliffs, NJ: Prentice-Hall, 1973.

Fomel, S. Seismic data decomposition into spectral components using regularized nonstationary autoregression. Geophysics 2013, 78, 069-76. 
Goupillaud, P.; Grossman, A.; Morlet, J. Cycle-octave and related transforms in seismic signal analysis. Geoexploration 1984, 23, 85-102.

Grossman, A.; Morlet, J. Decomposition of Hardy functions into square integrable wavelets of constant shape. SIAM J. Math. Anal. 1984, 15, 723-736.

Holmström, K.; Peterson, J. A review of the parameter estimation problem of fitting positive exponential sums to empirical data. Appl. Math. Comput. 2002, 126, 31-61.

Marple-Jr, S. Digital spectral analysis with applications; Englewood Cliffs, NJ: Prentice-Hall, 1987.

McDonough, R.; Higgins, W. Best least-squares representation of signals by exponentials. IEEE Transactions on Automatic Control 1968, 13, 408-412.

Mitrofanov, G.; Priimenko, V. Prony filtration of seismic data: theoretical background. Brazilian Journal of Geophysics 2011, 29, 703-722.

Mitrofanov, G.; Priimenko, V. Prony filtering of seismic data. Acta Geophysica 2015, 63, 652-678.

Osbourne, M.; Smyth, G. A modified Prony algorithm for exponential functional fitting. SIAM J. Sci. Comput. 1995, 16, 119-138.

Potts, D.; Tashe, M. Parameter estimation for exponential sums by approximate Prony method. Signal Process. 2010, 90, 1631-1642.

Prony, R. Essai expérimental et analytique Sur les lois de la Dilatabilité des fluides élastiques et sur celles de la Force expansive de la vapeur de l'eau et de la vapeur de l'alkool, à différentes températures. Journal de l'Ecole Polytechnique 1795, 1, 24-76.

Rodríguez, A.F.; de Santiago Rodrigo, L.; Guillén, L.L.; Ascariz, J.R.; Jiménez, J.M.; Boquete, L. Coding Prony's method in MatLab and applying it to biomedical signal filtering. BMC Bioinformatics 2018, 19, 214.

Soares Filho, D.; Priimenko, V.; Kremlev, A.; Misságia, R.; others. Processamento de Dado Sísmico com Técnica de Filtragem de Prony e Imageamento WCDP, 2003.

I.O.: Computational implementation of the Prony decomposition method, elaboration of the workflow, signal modeling, generation and analysis of the results. G.M. and V.P.: Development of mathematical basis, construction of the workflow and analysis of the results. J.F.: contributed significantly through support for the implementation of Python processing routines, applying object-oriented techniques and optimization to reduce computational cost. 